\title{
Pseudo-Analysis: measures of general conditional information
}

\author{
DORETTA VIVONA ${ }^{1,}$, MARIA DIVARI ${ }^{2}$ \\ 1 "Sapienza"-University of Rome, Department of Basic and Applied Sciences for Engeneering, 00161, Rome Italy \\ ${ }^{2}$ Retaired, Italy
}

\begin{tabular}{l} 
A R T I C L E I N F O \\
\hline Article history: \\
Received: 28 December, 2016 \\
Accepted: 06 February, 2017 \\
Online: 25 February, 2017 \\
\hline Keywords: \\
General information \\
Conditional information \\
Pseudo-operations \\
Independent property \\
Functional equations
\end{tabular}

\section{Introduction}

Since 1967 Kampé De Feriét and Forte introduced, by axiomatic way, the definition of measures $J$ for general information, where general means that $J$ is defined without probability [1].

Later, in [2], we introduced some particular family of crisp set $\left(\mathcal{N}, \mathcal{F}, \mathcal{I}_{\infty}, \mathcal{I}_{0}\right)$, in order to study the integration in information theory without probability. We have used them for the definition of measures of general conditional information [3] .

In this paper, we would continue the researches in the setting of Pseudo-Analysis, started in [4], by using pseudo-addition and pseudo-difference. In particular we shall study measures for general conditional information for crisp sets.

The properties of the form of conditional information have translated in a system of functional equations [5], for which we shall give a class of solutions.

Moreover, by using the property of $J$ independence we obtain another equation: we shall find the general solution through our previous result [6].

The paper is organize in the following way: in Sect. 2 we recall some preliminaires; in Sect. 3 we give the definition of general information conditioned by a variable event in pseudo-analysis. In Sect. 4 we con- sider the statement of the problem and we traslate the properties of the form of conditional information in a system of functional equations.

We shall distinguish two cases: general case and independent case. In this last case the definition of independence is given by using the pseudo-analysis. We shall show some classes of solutions in Sect. 5. Sect. 6 is devoted to the conclusions.

\section{Preliminary notations}

\subsection{Pseudo-operations}

We follow the Theory of Pseudo-Analysis introduced by E.Pap and his collegue [7], which consider the definition of pseudo operations. In particular, we shall use the pseudo-addition $\oplus$ and the pseudo-difference $\ominus$ : for knownledge about these pseudo-operations, we refer to [8].

Definition 2.1 The pseudo-addition $\oplus$ is a binary function

$$
\oplus:[0, M]^{2} \longrightarrow[0, M], M \in(0,+\infty]
$$

which is commutative, associative, strictly increasing with respect $\leq$, with 0 as neutral element.

\footnotetext{
${ }^{*}$ Corresponding Author Doretta Vivona, via A.Scarpa n.16, I-00161 Rome (Italy), tel.39-06-4976-6649, fax 39-06-4957-647 \& Emal: doretta.vivona@sbai.uniroma1.it
} 
We shall consider only particular operation $\oplus$ expressed by a function $g$, called generator function in the following way:

$$
u \oplus_{g} v=g^{-1}(g(u)+g(v)),
$$

where $g:[0, M] \longrightarrow[0,+\infty]$ and it is bijective, continuous and strictly increasing with $g(0)=0$ and $g(+\infty)=$ $+\infty$.

Definition 2.2 The pseudo-difference $\ominus$ is a mapping,

$$
\ominus:[0, M]^{2} \longrightarrow[0, M], M \in(0,+\infty],
$$

which we shall define through the same function $g$ :

$$
u \ominus_{g} v=g^{-1}(g(u)-g(v)), \quad u \geq v .
$$

\subsection{Measures of general information in classical analysis}

Following [1], let $X$ be an abstract space and $\mathcal{A}$ a $\sigma$-algebra of all subsets of $X$, such that $(X, \mathcal{A})$ is a measurable space.

Definition 2.3 Measure of the general information is a mapping

$$
J(\cdot): \mathcal{A} \rightarrow[0,+\infty]
$$

such that $\forall A_{1}, A_{2} \in \mathcal{A}$ :

- 2.3 1) $A_{1} \supset A_{2} \Rightarrow J\left(A_{1}\right) \leq J\left(A_{2}\right)$,

- 2.32) $J(\emptyset)=+\infty \quad, \quad J(X)=0$.

Moreover, we have the following [1]:

Definition 2.4 Given a subfamily $\mathcal{K} \subset \mathcal{A}$, two sets $K, K^{\prime} \in \mathcal{K}, K \neq K^{\prime}, K \cap K^{\prime} \neq \emptyset$ are called $J$-independent (i.e. independent with respect to $J$ ) if the couple $\left(K, K^{\prime}\right)$ satisfies the following:

$$
J\left(K \cap K^{\prime}\right)=J(K)+J\left(K^{\prime}\right) .
$$

From [2], assigned an information measure $J$, we have considered the family:

$$
\mathcal{I}_{+\infty}=\{\mathrm{F} \in \mathcal{A} / \mathrm{J}(\mathrm{F})=+\infty\} .
$$

The family (4) is not empty because it contains the empty set $\emptyset$ and all subsets $F^{\prime}$ of $F \in \mathcal{I}_{+\infty}$ :

$F \in \mathcal{I}_{+\infty}, \mathrm{J}(\mathrm{F})=+\infty, \forall \mathrm{F}^{\prime} \in \mathcal{A}, \mathrm{F}^{\prime} \subset \mathrm{F}, \mathrm{J}(\mathrm{F}) \geq \mathrm{J}\left(\mathrm{F}^{\prime}\right)=$ $+\infty \Longrightarrow \mathrm{F}^{\prime} \in \mathcal{I}_{+\infty}$.

$\mathcal{I}_{+\infty}$ is not an filter [9] because it is not stable with respect to the intersection between fuzzy sets.

Given the family $\mathcal{H}=\mathcal{A}-\mathcal{I}_{+\infty}$ we recall from [3],

Definition 2.5 The measure of general conditional information of any set $A \in \mathcal{A}$ conditioned by a fixed $H \in \mathcal{H}$, $(J(A \mid H))$ is a mapping

$$
J(\cdot \mid H): \mathcal{A} \rightarrow[0,+\infty]
$$

such that

- 2.5 1) $A^{\prime} \supset A \Longrightarrow J\left(A^{\prime} \mid H\right) \leq J(A \mid H), \quad \forall A, A^{\prime} \in \mathcal{A}$,
- 2.5 2) $J(\emptyset \mid H)=+\infty$,

- 2.53) $J(X \mid H)=0$.

Moreover, from [2], we have the following:

Definition 2.6 Given a subfamily $\mathcal{K} \subset \mathcal{A}$, two sets $K, K^{\prime} \in \mathcal{K}, K \neq K^{\prime}, K \cap K^{\prime} \neq \emptyset$ are called $J$-conditional independent (i.e. conditioned by a fixed event $H \in \mathcal{H}$ if the couple $\left(K, K^{\prime}\right)$ satisfies the following condition:

$$
J\left(\left(K \cap K^{\prime}\right) \mid H\right)=J(K \mid H)+J\left(K^{\prime} \mid H\right) .
$$

\section{Pseudo-analysis: measures of general conditional information}

In [4], for the first time, we have introduced the definition of $J$ - independence property in the setting of pseudo-analysis, and we have used it to find the information of the union of two sets $A, A^{\prime} \in \mathcal{A}: J\left(A \cup A^{\prime}\right)$. From now on we consider a pseudo-addition $\oplus_{g}$ generated by a function $g$ as in 11 .

Definition 3.1 Given a subfamily $\mathcal{K} \subset \mathcal{A}$, and a pseudo-addition $\oplus_{g}$, two sets $K, K^{\prime} \in \mathcal{K}$, are called $J$ independent in pseudo-analysis if the couple $\left(K, K^{\prime}\right)$ satisfies the following:

$$
J\left(K \cap K^{\prime}\right)=J(K) \oplus_{g} J\left(K^{\prime}\right), \quad K \neq K^{\prime}, K \cap K^{\prime} \neq \emptyset .
$$

In pseudo-analysis, for the general conditional information, we shall replace the common addition in (3) with the pseudo-addition $\oplus_{g}$, so we shall propose the following:

Definition 3.2 Given a subfamily $\mathcal{K} \subset \mathcal{A}$, two sets $K, K^{\prime} \in \mathcal{K}$ are called $J$ - conditional independent in pseudo-analysis if the couple $\left(K, K^{\prime}\right)$ satisfies the following property:

$$
\begin{gathered}
J\left(\left(K \cap K^{\prime}\right) \mid H\right)=J(K \mid H) \oplus_{g} J\left(K^{\prime} \mid H\right), \\
K \neq K^{\prime}, K \cap K^{\prime} \neq \emptyset .
\end{gathered}
$$

In [10] we have generalize the property of $J$-independence.

\section{Statment of the problem: the function $\Phi$}

In this paragraph, fixed an information $J$, we would look for the measure of general conditional information of any set $A \in \mathcal{A}$ conditioned by a fixed $H \in \mathcal{H}$,

$$
J^{*}(A \mid H)
$$

as a function $\Phi$ which depends only on $J(A \cap H)$ and $J(H)$. We suppose that the function $\Phi$ is continuous

$$
\Phi: T \longrightarrow(0,+\infty],
$$

where $T=\{(x, y) / x, y \in[0,+\infty], x \geq y: \exists A \in \mathcal{A}, H \in$ $\mathcal{H}, x=J(A \cap H), y=J(H)\}$ and

$$
J^{*}(A \mid H)=\Phi(J(A \cap H), J(H)) .
$$




\subsection{General case}

The conditions 2.51$)-(2.53)$ become:

$$
\left\{\begin{aligned}
&(\text { E1) } \Phi\left(J\left(A^{\prime} \cap H\right), J(H)\right) \leq \Phi(J(A \cap H), J(H)), \\
& \forall A, A^{\prime} \in \mathcal{A}, A^{\prime} \supset A \\
&(\text { E2) } \Phi(J(\emptyset), J(H))=+\infty \\
&(\text { E3) } \Phi(J(H), J(H))=0 .
\end{aligned}\right.
$$

Setting $J(A \cap H)=x, J\left(A^{\prime} \cap H\right)=x^{\prime}, J(H)=y, x, x^{\prime}, y \in$ $[0,+\infty], x^{\prime} \geq y, x \geq y$ the equations $(E 1)-(E 3)$ get the following form:

$$
\left\{\begin{array}{l}
\left(\text { e1) } \Phi\left(x^{\prime}, y\right) \leq \Phi(x, y), \quad x^{\prime} \leq x, x^{\prime} \geq y, x \geq y,\right. \\
\text { (e2) } \Phi(+\infty, y)=+\infty \\
\text { (e3) } \Phi(y, y)=0
\end{array}\right.
$$

\subsection{Independent case}

In this paragraph, we shall consider $J$ - independent sets in pseudo-analysis.

We suppose that there exist two sets $K, K^{\prime} \in \mathcal{K}, K \neq$ $K^{\prime}, K \cap K^{\prime} \neq \emptyset$, which are $J$ - independent in pseudoanalysis in the sense of (6). From (7), and taking into account (8), it is

$$
\begin{aligned}
\left.J^{*}\left(\left(K \cap K^{\prime}\right) \mid H\right)=\Phi\left(J\left(K \cap K^{\prime}\right) \cap H\right), J(H)\right)= \\
=\Phi\left(J\left(K \cap H \cap K^{\prime} \cap H\right), J(H)\right)= \\
=\Phi\left(J(K \cap H) \oplus_{g} J\left(K^{\prime} \cap H\right), J(H)\right),
\end{aligned}
$$

On the other hand, by (8),

$$
\begin{gathered}
J^{*}(K \mid H)=\Phi(J(K \cap H), J(H)), \\
J^{*}\left(K^{\prime} \mid H\right)=\Phi\left(J\left(K^{\prime} \cap H\right), J(H)\right),
\end{gathered}
$$

Then, we obtain the condition of $J$-independence in pseudo-analysis:

$$
\begin{gathered}
\Phi\left(J(K \cap H) \oplus_{g} J\left(K^{\prime} \cap H\right), J(H)\right)= \\
\Phi(J(K \cap H), J(H)) \oplus_{g} \Phi\left(J\left(K^{\prime} \cap H\right), J(H)\right), \\
K, K^{\prime} \in \mathcal{K}, K \neq K^{\prime}, K \cap K^{\prime} \neq \emptyset .
\end{gathered}
$$

Setting $J(K \cap H)=t, J\left(K^{\prime} \cap H\right)=t^{\prime}, t, t^{\prime} \in[0,+\infty], t \neq$ $t^{\prime}, t \geq y, t^{\prime} \geq y$ with $J(H)=y$, the 10 becomes

$$
\text { (e4) } \begin{gathered}
\Phi\left(t \oplus_{g} t^{\prime}, y\right)=\Phi(t, y) \oplus_{g} \Phi\left(t^{\prime}, y\right), \quad t, t^{\prime} \in[0,+\infty], \\
t \neq t^{\prime}, t \geq y, t^{\prime} \geq y .
\end{gathered}
$$

\section{Solutions of the problem}

Now, we are giving some solutions of the problem, distinguishing two previous cases.

\subsection{General case}

Proposition 5.1 A class of continuous solutions of the system $(\mathbf{e} \mathbf{1})-(\mathbf{e} 3)$ is

$$
\Phi_{\rho}(x, y)=\rho^{-1}\left(\rho(x) \ominus_{g} \rho(y)\right),
$$

where $\rho:[0,+\infty] \longrightarrow[0,+\infty]$ is any bijective, continuous, strictly increasing function, with $\rho(0)=0, \rho(+\infty)=$ $+\infty$ and $\ominus$ is defined in (2).

Proof. The condition (e1) is satisfied as the composition of two increasing functions $\rho$ and $g$. The conditions $(e 2)$ and $(e 3)$ are verified by the values $g(0)=$ $\rho(0)=0$ and $\rho(+\infty)=g(+\infty)=+\infty$. Moreover $\rho$ is continuous as the generator function $g$.

Proposition 5.2 Another class of continuous solutions of the system $(\mathbf{e} \mathbf{1})-(\mathbf{e} \mathbf{3})$ is

$$
\Phi_{\mu}(x, y)=x \ominus_{\mu} y, \quad \mu=g \cdot m
$$

where $\mu$ is the product of the generator function $g$ of the operation $\ominus_{g}$ given by (2) and $m$ is any function as in (12).

Proof. Let $m$ be a particular function solution of the system $(e 1)-(e 3)$ as in Prop.[5.1], which defines a pseudo-addition $\oplus_{m}$. From (2) and (10), it is

$$
\Phi_{m}(x, y)=m^{-1}\left(m(x) \ominus_{g} m(y)\right)=
$$

$$
\begin{gathered}
=m^{-1}\left\{g^{-1}((g(m(x))+(g(m)(y)))\}=\right. \\
=\mu^{-1}(\mu(x)+\mu(y))=x \ominus_{\mu} y,
\end{gathered}
$$

where $\mu=g \cdot m \Longleftrightarrow \mu^{-1}=m^{-1} \cdot g^{-1}$

By the properties of $g$ and $m$ the solutions are continuous.

Any function $\rho$ in Prop.[5.1], in general, doesn't define a pseudo-addition of the kind $\oplus_{g}$ as in (1) because it is not commutative, neither associative. For this reason the Prop.[5.2] is not a consequence of Prop.[5.1]. 


\subsection{Independent case}

Now, we rewrite the equation (e4):

$$
\begin{gathered}
\text { (e4) } \Phi\left(t \oplus_{g} t^{\prime}, y\right)=\Phi(t, y) \oplus_{g} \Phi\left(t^{\prime}, y\right), \\
t, t^{\prime} \in[0,+\infty], t \neq t^{\prime}, t \geq y, t^{\prime} \geq y .
\end{gathered}
$$

Fixed $y=y^{*}$, the condition (e4) is

$$
\Phi\left(t \oplus_{g} t^{\prime}, y^{*}\right)=\Phi\left(t, y^{*}\right) \oplus_{g} \Phi\left(t^{\prime}, y^{*}\right),
$$

setting

$$
\Phi\left(t, y^{*}\right)=\Psi(t),
$$

the equation (15) becomes

$$
\Psi\left(t \oplus_{g} t^{\prime}\right)=\Psi(t) \oplus_{g} \Psi\left(t^{\prime}\right) .
$$

The equation (17) is a particular case of a general Cauchy equation

$$
F(x \oplus y)=F(x) \oplus F(y)
$$

on suitable hypothesys on the function $F$, when $\oplus$ is any pseudo-addition not necessary expressed by a generator function $g$. The equation (17) has been solved by Benvenuti and the authors in [6] in many general cases.

In particular, when this pseudo-addition is generated by function $g$, we found all continuous solutions. Here, we neglect trivial solutions and we consider only the most meanigfull solution. We recall the result from [6]:

Theorem 5.3 The solution of the general Cauchy equation

$$
F\left(x \oplus_{g} y\right)=F(x) \oplus_{g} F(y),
$$

under suitable hypothesys on $F$, when the operation $\oplus_{g}$ is defined by a generator function $g$

$$
u \oplus_{g} v=g^{-1}(g(u)+g(v))
$$

is (really, we should say of a class of solution depending on a parameter $\lambda$ ) the following continuous function

$$
F_{\lambda}(x)=g^{-1}(\lambda \cdot g(x)), \lambda \in(0,+\infty),
$$

with $g$ the generator function of the pseudo-addition (2).

By using the previuos result, the class of solution of 16$)$ is

$$
\Psi(t)=g^{-1}(\lambda \cdot g(t)), \lambda \in(0,+\infty),
$$

with $g$ the generator function of the pseudo-addition (2).

Now, we can go back to our original problem concerning the $J$ - independence property in pseudoanalysis and we are ready to give the main theorem.

Let $\mathcal{L}$ be any family of continuous function $\Lambda$ : $(0,+\infty) \rightarrow(0,+\infty):$

$$
\mathcal{L}=\{\Lambda:(0,+\infty) \rightarrow(0,+\infty), \text { continuous }\} .
$$

Theorem 5.4 The class of continuous solutions of the equation

(e4) $\Phi\left(t \oplus_{g} t^{\prime}, y\right)=\Phi(t, y) \oplus_{g} \Phi\left(t^{\prime}, y\right)$,

$$
t, t^{\prime} \in[0,+\infty], t \neq t^{\prime}, t \geq y, t^{\prime} \geq y
$$

is the family, depending on any element $\lambda(y)$ of $\mathcal{L}$,

$$
\Phi_{\Lambda(y)}(t)=g^{-1}(\Lambda(y) \cdot g(t)) .
$$

Proof. Now, fixed any function $\Lambda(y) \in \mathcal{L}$, we are verifying that $(22)$ is solution of (e4):

$$
\begin{gathered}
\Phi\left(t \oplus_{g} t^{\prime}, y\right)=\Phi_{\lambda(y)}\left(t \oplus t^{\prime}\right)=g^{-1}\left(\Lambda(y) \cdot g\left(t \oplus t^{\prime}\right)\right)= \\
=g^{-1}\left(\Lambda(y) \cdot g\left\{g^{-1}\left[g(t)+g\left(t^{\prime}\right)\right]\right\}\right)= \\
=g^{-1}\left(\Lambda(y) \cdot\left[g(t)+g\left(t^{\prime}\right)\right]\right)= \\
=g^{-1}\left(\Lambda(y) \cdot g(t)+\Lambda(y) \cdot g\left(t^{\prime}\right)\right)= \\
=g^{-1}\left(g g^{-1}[\Lambda(y) \cdot g(t)]+g g^{-1}\left[\Lambda(y) \cdot g\left(t^{\prime}\right)\right]\right)= \\
=g^{-1}\left(g\left\{g^{-1}[\Lambda(y) \cdot g(t)]\right\}+g\left\{g^{-1}\left[\Lambda(y) \cdot g\left(t^{\prime}\right)\right]\right\}\right)= \\
=g^{-1}\left(g\left\{\Phi_{\Lambda(y)} \cdot g(t)\right\}+g\left\{\Phi_{\Lambda(y)} \cdot g\left(t^{\prime}\right)\right\}\right)= \\
=\left(\Phi_{\Lambda(y)}(t)\right) \oplus_{g}\left(\Phi_{\Lambda(y)}\left(t^{\prime}\right)\right)=\Phi(t, y) \oplus_{g} \Phi\left(t^{\prime}, y\right) .
\end{gathered}
$$

It is easy to see that any function 22 is continuous.

Proposition 5.5 The function $\Phi_{\Lambda(y)}(t)$ given by 22) is strictly increasing with respect to the variable $t$.

Proof. The monotonicity of the function $\Phi_{\Lambda(y)}(t)$ doesn't depend on the variable $y$; moreover $\Lambda(y)$ is positive. Then,

$$
\forall t \leq t^{*} \text { and } \forall \Lambda(y) \in \mathcal{L}, \Phi_{\Lambda(y)}(t) \leq \Phi_{\Lambda(y)}\left(t^{\prime}\right) .
$$

As conseguence of Theorem [5.4] and of Proposition [5.5], we get the following

Theorem 5.6 The only solution of the equations

(e1) $\Phi\left(x^{\prime}, y\right) \leq \Phi(x, y) \quad x^{\prime} \leq x, x^{\prime} \geq y, x \geq y$

(e4) $\Phi\left(t \oplus_{g} t^{\prime}, y\right)=\Phi(t, y) \oplus_{g} \Phi\left(t^{\prime}, y\right)$,

$$
t, t^{\prime} \in[0,+\infty], t \neq t^{\prime}, t \geq y, t^{\prime} \geq y .
$$

is the family, depending on any element of $\mathcal{L}$, given by (21),

$$
\Phi_{\Lambda(y)}(t)=g^{-1}(\Lambda(y) \cdot g(t)) .
$$

It is easy to see that the conditions (e2) and (e3) are not compatible with the independent property. 


\section{Conclusion}

In this paper, given a measure of general information $J$, we have defined the measure of general conditional information $J^{*}(A \mid H)$ of any set $A \in \mathcal{A}$ conditioned to $H \in \mathcal{H}$.

Moreover, we have considered $J^{*}(A \mid H)$ depending only on $J(A \cap H)$ and $J(H)$ through a function $\Phi$. The properties of this $J^{*}(A \mid H)$ are translated in a system of functional equations.

In order to look for solutions of the system, we distinguish two cases: the first concerning monotonicity and particular values of $J^{*}(A \mid H)$, system (e1)-(e3), the second one related to monotonicity and independence property, equations (e1) and (e4).

\section{I-General case: system (e1)-(e3)}

Some classes of the measure of general conditional information are: from $(12)$

$$
J_{\rho}^{*}(A \mid H)=\rho^{-1}\left(\rho(J(A \cap H)) \ominus_{g} \rho(J(H))\right),
$$

where $\rho:[0,+\infty] \longrightarrow[0,+\infty]$ is any bijective, continuous, strictly increasing function, with $\rho(0)=$ $0, \rho(+\infty)=+\infty$

and from 13

$$
\left.J_{\mu}^{*}(A \mid H)=J(A \cap H)\right) \ominus_{\mu} J(H),
$$

where $\mu$ is the product of the generator function $g$ of the operation $\ominus_{g}$ given by (2) and $m$ is any function as in (1).

\section{II-Independent case: equations (e1) and (e4)}

From (23) one and only one class of solutions is:

$$
J^{*}(A \mid H)=g^{-1}(\Lambda(J(H)) \cdot g(J(A \cap H))),
$$

depending on a class of functions $\Lambda \in \mathcal{L}$, where $\in \mathcal{L}$ is defined in 21.

Conflict of Interest The authors declare no conflict of interest.
Acknowledgment This research is in the framework of GNFM (Gruppo Nazionale per la Fisica Matematica) del MIUR (Ministero Italiano per l’Universitá e la Ricerca), ITALY.

\section{References}

1. J.Kampe De Feriet, B.Forte, Information et Probabilite applications" Compt.Rand.Ac.Sc.Paris, 265, 110-114, 142-146, 350-353, 1967.

2. D.Vivona, Information Theory and Fuzzy Integrals: along the way paved by Pietro Benvenuti, 10th International Conference on Information Processing and Management of Uncertainty in Knowledge-based System (IPMU), Perugia Italy, 2004.

3. D.Vivona - M.Divari, On a conditional information for fuzzy sets, International Summer School on Aggregation Operators and their applications (AGOP), Lugano Switzerland, 2005.

4. D.Vivona - M.Divari, Pseudo-Analysis: some measures of general information, IEEE 14th International Symposium on Intelligent Systems and Informatics (SISY), Subotica Serbia, 2016.

5. J. Aczel, Lectures on functional equations and their applications, New Jork, Academic Press, 1966.

6. P.Benvenuti - R.Mesiar - D.Vivona, The cauchy equation on I-semigroup, Aequ.Math. 63, 2002.

7. E. Pap, Null-additive Set Functions Dordrecht. Kluver Academic Publishers, 1995.

8. P.Benvenuti - R.Mesiar - D.Vivona, Monotone set-functions-based integrals, Handbook in Measure Theory, Elsevier Ed. 2002.

9. K.Yosida, Functional analysis, Springer, 1995.

10. D.Vivona - M.Divari, An Independence property for General Information, Natural Sciences 8 2016, dx.doi.org/10.4236/ns.2016.82008. 